25 September 1997, to be published in PHYSICA E

\title{
Semiclassical Approach to Orbital Magnetism of Interacting Diffusive Quantum Systems
}

\author{
D. Ullmo, ${ }^{(1,2)}$ K. Richter, ${ }^{(3)}$ H.U. Baranger, ${ }^{(1)}$ F. von Oppen, ${ }^{(4)}$ and R.A. Jalabert ${ }^{(5)}$ \\ (1) Bell Laboratories-Lucent Technologies, 700 Mountain Ave., Murray Hill NJ 07974 \\ (2) Division de Physique Théorique, Institut de Physique Nucléaire, 91406 Orsay Cedex, France \\ (3) Max-Planck-Institut für Physik komplexer Systeme, Nöthnitzer Str. 38, 01187 Dresden Germany \\ (4) Department of Condensed Matter Physics, Weizmann Institute, 76100 Rehovot, Israel \\ (5) Université Louis Pasteur, IPCMS-GEMME, 23 rue du Loess, 67037 Strasbourg Cedex, France
}

\begin{abstract}
We study interaction effects on the orbital magnetism of diffusive mesoscopic quantum systems. By combining many-body perturbation theory with semiclassical techniques, we show that the interaction contribution to the ensemble averaged quantum thermodynamic potential can be reduced to an essentially classical operator. We compute the magnetic response of disordered rings and dots for diffusive classical dynamics. Our semiclassical approach reproduces the results of previous diagrammatic quantum calculations.
\end{abstract}

\section{A. Introduction}

The interplay of disorder and interactions in mesoscopic systems has attracted considerable attention [1]. Interaction effects on transport through small quantum dots [2] as well as on thermodynamic properties like persistent currents and orbital magnetism are of present interest. In the latter case, the unexpectedly large measured persistent current of small metal rings [3] [5] pointed towards the importance of such interaction effects and motivated a large number of theoretical approaches [6].

For the description of thermodynamic quantities, semiclassical expansions have proven particularly useful, both within the independent-particle model [79] and for interaction effects [10,11]. These studies established a close relation between the classical dynamics and the quantum-mechanical magnetic response. In particular, studies of ballistic systems showed that the quantum thermodynamic properties are sensitive to whether the classical dynamics is regular or chaotic [8,9,11].

In this paper we apply these semiclassical techniques to the orbital magnetism of interacting systems whose non-interacting classical dynamics is diffusive. Specifically, we present 
semiclassical derivations of the interaction contributions to the persistent current of metal rings and to the susceptibility of singly-connected two-dimensional diffusive systems. We recover results obtained previously by quantum diagrammatic calculations [12 [17], showing that the semiclassical approach is on the same level of approximation. By semiclassically evaluating the relevant diagrams appearing in the many-body perturbation series for the thermodynamic potential, we express the latter in terms of an essentially classical operator. This expression provides a convenient starting point for further calculations. Moreover, by making the connection with the classical dynamics, it provides a physically intuitive picture of the interplay between disorder and interaction.

\section{B. Diagrammatic perturbation theory}

We are interested in the orbital magnetism of a mesoscopic quantum system subject to an external magnetic field $B$. While the magnetic response of a singly-connected system is usually measured in terms of its susceptibility $\chi$, the magnetic moment of a ring-type structure threaded by a flux $\phi=B A$ (where $A$ is the enclosed area) is usually described by the related persistent current $I$. Both are given in terms of the thermodynamic potential $\Omega$ as ( $V$ being the area (volume) of the structure)

$$
I \equiv-c \frac{\partial \Omega}{\partial \phi} \quad ; \quad \chi \equiv-\frac{1}{V} \frac{\partial^{2} \Omega}{\partial B^{2}} .
$$

To calculate the interaction contribution to the magnetic response, the high-density expansion (RPA) of the thermodynamic potential [18] has to be extended by including interaction corrections from diagrams with the Cooper-channel. This was originally performed in the context of superconducting fluctuations and then applied to disordered normal metals [12 17. Such expansions usually yield reliable results even beyond the high density limit, if the relevant sets of terms are properly resummed. The relevant Cooper-like diagrams are shown in Fig. 1. The screened Coulomb interaction (wavy lines) can be treated as local 14, 15: $U\left(\mathbf{r}-\mathbf{r}^{\prime}\right)=\lambda_{0} N(0)^{-1} \delta\left(\mathbf{r}-\mathbf{r}^{\prime}\right)$. Here, $N(0)$ denotes the density of states and the bookkeeping index $\lambda_{0}=1$ identifies the order of perturbation. For the local interaction, direct and exchange term are equivalent up to a factor of $(-2)$ due to the spin sums and the different number of fermion loops. The corresponding perturbation expansion for this interaction contribution $\Omega$ to the thermodynamic potential, which yields the magnetic response, can be formally expressed as [13, 14]

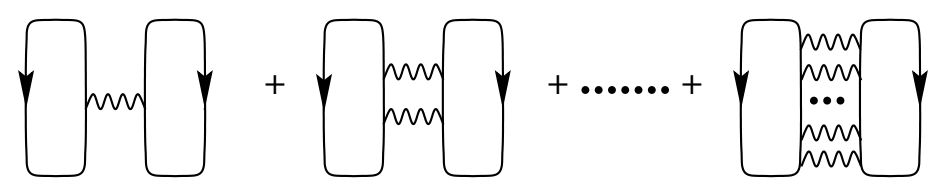

FIG. 1. Leading Cooper-channel diagrams for the interaction contribution to the thermodynamic potential. 


$$
\begin{aligned}
\Omega & =-\frac{1}{\beta} \sum_{n=1}^{\infty} \frac{\left(-\lambda_{0}\right)^{n}}{n} \sum_{\omega} \int d \mathbf{r}_{1} \ldots d \mathbf{r}_{n} \Sigma_{\mathbf{r}_{1}, \mathbf{r}_{2}}(\omega) \ldots \Sigma_{\mathbf{r}_{n}, \mathbf{r}_{1}}(\omega) \\
& =\frac{1}{\beta} \sum_{\omega} \operatorname{Tr}\left\{\ln \left[1+\lambda_{0} \hat{\Sigma}(\omega)\right]\right\} .
\end{aligned}
$$

Here, $\omega$ denotes the bosonic Matsubara frequencies $\omega=2 \tilde{n} \pi / \beta$ with $\beta=1 / k T$. The particleparticle propagator $\hat{\Sigma}(\omega)$ is expressed (in position representation) in terms of products of finite-temperature Green functions as [18]

$$
\Sigma_{\mathbf{r}, \mathbf{r}^{\prime}}(\omega)=\frac{1}{\beta N(0)} \sum_{\epsilon}^{E_{F}} \mathcal{G}_{\mathbf{r}, \mathbf{r}^{\prime}}(\epsilon) \mathcal{G}_{\mathbf{r}, \mathbf{r}^{\prime}}(\omega-\epsilon) .
$$

Here, the sum runs over the fermionic Matsubara frequencies $\epsilon=(2 n+1) \pi / \beta$. The shortlength (high-frequency) behavior is included in the screened interaction, thus requiring a cutoff of the frequency sums at the Fermi energy $E_{F}$ [14. The straight lines in Fig. 1 represent finite-temperature Green functions of the non-interacting system. They are of the form

$$
\mathcal{G}_{\mathbf{r}, \mathbf{r}^{\prime}}(\epsilon)=\theta(\epsilon) G_{\mathbf{r}, \mathbf{r}^{\prime}}^{R}\left(E_{F}+i \epsilon\right)+\theta(-\epsilon) G_{\mathbf{r}, \mathbf{r}^{\prime}}^{A}\left(E_{F}+i \epsilon\right)
$$

in terms of the retarded and advanced Green functions $G^{R, A}$ which are related by $G_{\mathbf{r}, \mathbf{r}^{\prime}}^{A}(E)=$ $\left[G_{\mathbf{r}^{\prime}, \mathbf{r}}^{R}\left(E^{*}\right)\right]^{*}$. For diffusive systems, they include the presence of the disorder potential.

\section{Semiclassical formalism}

Both in ballistic and diffusive samples, the Fermi wavelength $\lambda_{F}$ is often the shortest lengthscale. It is in this situation that we can apply semiclassical techniques to compute $\Sigma_{\mathbf{r}, \mathbf{r}^{\prime}}(\omega)$. Here, we will moreover assume that the magnetic field $B$ is classically weak, i.e., that the cyclotron radius $R_{c} \gg \min \{l, L\}$ (with $l$ the elastic mean free path and $L$ the system size).

Semiclassically, the retarded Green function is represented as a sum of contributions $G_{\mathbf{r}, \mathbf{r}^{\prime}}^{R ; j}$ over all classical paths $j$ from $\mathbf{r}$ to $\mathbf{r}^{\prime}[19$,

$$
G_{\mathbf{r}, \mathbf{r}^{\prime}}^{R}(E) \simeq \sum_{j: \mathbf{r} \rightarrow \mathbf{r}^{\prime}} D_{j} e^{i S_{j} / \hbar-i \pi \nu_{j} / 2}
$$

Here $S_{j}=\int_{\mathbf{r}}^{\mathbf{r}^{\prime}} \mathbf{p} \cdot d \mathbf{r}$ is the classical action of trajectory $j$. The prefactor $D_{j}$ includes the classical phase space density $\left[D_{j}=\left(1 / \sqrt{2 \pi(i \hbar)^{3} \dot{x} \dot{x}^{\prime}}\right)\left|\partial^{2} S_{j} / \partial y \partial y^{\prime}\right|^{1 / 2}\right.$ in two dimensions]. $\nu_{j}$ is a Maslov index. The semiclassical approximation makes the temperature and magnetic-field dependences of the finite-temperature Green function transparent. Employing $\left(\partial S_{j} / \partial E\right)=t_{j}$ and $\left(\partial S_{j} / \partial B\right)=(e / c) A_{j}$, where $t_{j}$ and $A_{j}$ are the traversal time and area, one finds

$$
G_{\mathbf{r}, \mathbf{r}^{\prime}}^{R ; j}\left(E_{F}+i \epsilon, B\right) \simeq G_{\mathbf{r}, \mathbf{r}^{\prime}}^{R ; j}\left(E_{F}, B=0\right) \times \exp \left[-\epsilon t_{j} / \hbar\right] \times \exp \left[i 2 \pi B A_{j} / \phi_{0}\right]
$$

where $\phi_{0}=h c / e$ is the flux quantum. Note that temperature exponentially suppresses the contributions of long paths to each Green function. 
Semiclassically, the particle-particle propagator $\Sigma_{\mathbf{r}, \mathbf{r}^{\prime}}(\omega)$ is then represented as a sum over pairs of paths between $\mathbf{r}$ and $\mathbf{r}^{\prime}$. Off-diagonal pairs (of different paths) generally contain highly oscillatory contributions which do not survive an ensemble (disorder) average. (There can be exceptions as discussed in Ref. [11].) On the other hand, the diagonal pairing of each orbit $j$ with its time reverse persists upon averaging since their dynamical phases $\exp \left[i S_{j}(B=0) / \hbar\right]$ cancel while retaining a magnetic-field dependence. A more detailed semiclassical analysis [1] shows that the Cooper series in Fig. 1 contains the magnetic-field sensitive contribution to $\Omega$ which is leading order in $\hbar$.

Using Eqs. (4), (5) and (6) in Eq. (3) and performing the Matsubara sum yields for the diagonal part of $\hat{\Sigma}$

$$
\Sigma_{\mathbf{r}, \mathbf{r}^{\prime}}^{(D)}(\omega) \simeq \frac{\hbar}{\pi N(0)} \sum_{j: \mathbf{r} \rightarrow \mathbf{r}^{\prime}}^{L_{j}>\Lambda_{0}}\left|D_{j}\right|^{2} \frac{R\left(2 t_{j} / t_{T}\right)}{t_{j}} \times \exp \left[\frac{i 4 \pi B A_{j}}{\phi_{0}}\right] \times \exp \left[-\frac{\omega t_{j}}{\hbar}\right] .
$$

The sum runs over all trajectories longer than the cutoff $\Lambda_{0}=\lambda_{F} / \pi$ [corresponding to the upper bound $E_{F}$ on the Matsubara sum in Eq. (3)]. The temperature dependence in Eq. (7) enters through the function $R(x)=x / \sinh (x)$ introducing the time scale

$$
t_{T}=\frac{\hbar \beta}{\pi}
$$

and the related length scale $L_{T}=v_{F} t_{T}$, with $v_{F}$ being the Fermi velocity. This semiclassical framework allows us to reduce the original quantum problem to $\Sigma^{(D)}$ which no longer exhibits variations on the quantum scale $\lambda_{F}$ but only on classical scales. We emphasize that the representation (17) of $\Sigma^{(D)}$ is rather general since we have not yet made any assumption about the classical dynamics of the system. In particular, it applies to both diffusive and ballistic systems. On the basis of Eq. (7), we have recently studied interaction effects in ballistic quantum dots 11. Specifically, we show that the interaction induced orbital magnetism scales differently for systems with regular and chaotic non-interacting classical counterparts.

Here, we focus on diffusive systems for which it is useful to relate $\Sigma^{(D)}$ to classical probabilities satisfying the diffusion equation. To this end we introduce an additional time integration in Eq. (7) and make use of the relation [7]

$$
\frac{1}{2 \pi^{2}} \sum_{j: \mathbf{r} \rightarrow \mathbf{r}^{\prime}}\left|D_{j}\right|^{2} \delta\left(t-t_{j}\right)=\frac{N(0)}{2 \pi \hbar} P\left(\mathbf{r}, \mathbf{r}^{\prime} ; t\right)
$$

between the weights $\left|D_{j}\right|^{2}$ and the classical probability $P\left(\mathbf{r}, \mathbf{r}^{\prime} ; t\right)$ to propagate from $\mathbf{r}$ to $\mathbf{r}^{\prime}$ in time $t$.

An $n$-th order contribution to $\Omega$ in Eq. (2) then contains expressions for the joint return probability $P\left(\mathbf{r}_{1}, \ldots, \mathbf{r}_{n}, \mathbf{r}_{1} ; t_{1}, \ldots, t_{n} \mid A\right)$ to visit the points $\mathbf{r}_{i}$ (with $t_{i}$ being the time between $\mathbf{r}_{i}$ and $\mathbf{r}_{i+1}$ ) under the condition that the enclosed area is $A$. In diffusive systems, the probability is multiplicative, namely $\int d \mathbf{r}_{1} \ldots d \mathbf{r}_{n} P\left(\mathbf{r}_{1}, \ldots, \mathbf{r}_{n}, \mathbf{r}_{1} ; t_{1}, \ldots, t_{n} \mid A\right)=\int d \mathbf{r} P\left(\mathbf{r}, \mathbf{r} ; t_{\text {tot }} \mid A\right)$ with $t_{t o t}=\sum t_{i}$. The contribution to $\Omega$ in Eq. (2) from the diagonal terms $\Sigma^{(D)}$ then yields 


$$
\Omega^{(D)}=\sum_{n} \Omega_{n}^{(D)}=\frac{1}{\beta} \int d \mathbf{r} \int d t \operatorname{coth}\left(\frac{t}{t_{T}}\right) K(t) \mathcal{A}(\mathbf{r}, t ; B)
$$

where the $\operatorname{coth}\left(t / t_{T}\right)$ arises from the $\omega$-sum in Eq. (2) and

$$
\begin{aligned}
K(t) & \equiv \sum_{n} K_{n}(t)=-\sum_{n} \frac{\left(-\lambda_{0}\right)^{n}}{n}\left\{\int \prod_{i=1}^{n}\left[\frac{d t_{i} R\left(2 t_{i} / t_{T}\right)}{t_{i}}\right] \delta\left(t-t_{t o t}\right)\right\} \\
\mathcal{A}(\mathbf{r}, t ; B) & \equiv \int d A \cos \left(\frac{4 \pi B A}{\phi_{0}}\right) P(\mathbf{r}, \mathbf{r} ; t \mid A) .
\end{aligned}
$$

$K(t)$ accounts for temperature effects while $\mathcal{A}$ contains the field dependence and the classical return probability. Eqs. (10)-(12) are a general and convenient starting point to compute the orbital response of disordered systems.

\section{Diffusive rings}

We start with the computation of the first-order interaction contribution, $\Omega_{1}^{(D)}$, to illustrate the main ideas. Consider a (thin) disordered ring of width $b$, crossection $\sigma$ and circumference $L$. For $L \gg l, b$ the motion of particles around the ring effectively follows a law for one-dimensional diffusion. Since the area enclosed is given in terms of the number $m$ of windings around the ring, one has

$$
P(\mathbf{r}, \mathbf{r} ; t \mid A)=\sum_{m=-\infty}^{+\infty} \frac{1}{\sigma} \frac{1}{\sqrt{4 \pi D t}} \exp \left(-\frac{m^{2} L^{2}}{4 D t}\right) \delta\left(A-\frac{m L^{2}}{4 \pi}\right),
$$

where $D=v_{F} l / d$ is the diffusion constant (in $d$ dimensions). Because of the disorder average the classical return probability does not depend on $\mathbf{r}$. In first order we have

$$
K_{1}(t)=\lambda_{0} R\left(2 t / t_{T}\right) / t
$$

Combining this with the coth function in Eq. (10) we find

$$
\Omega_{1}^{(D)}=\lambda_{0} \frac{L \hbar}{\pi} \sum_{m=-\infty}^{+\infty} \cos \left(\frac{4 \pi m \phi}{\phi_{0}}\right) g_{m}(T)
$$

with

$$
g_{m}(T)=\int_{0}^{\infty} d t \frac{R^{2}\left(t / t_{T}\right)}{t^{2}} \frac{\exp \left[-(m L)^{2} /(4 D t)\right]}{\sqrt{4 \pi D t}} .
$$

Taking the derivative with respect to the flux, we recover the first-order interaction contribution to the persistent current, first obtained in [15] by purely diagrammatic techniques,

$$
I_{1}=\lambda_{0} \frac{2 L e}{\pi} \sum_{m=-\infty}^{+\infty} m \sin \left(\frac{4 \pi m \phi}{\phi_{0}}\right) g_{m}(T) .
$$

Semiclassically, this first order result was already derived by Montambaux [10]. 
In addition, our semiclassical approach allows us to obtain the renormalization of the coupling constant [12 16] due to the higher-order diagrams of the Cooper series. Including these diagrams amounts to using the full kernel $K(t)$ in Eq. (10) instead of $K_{1}(t)$. Introducing the Laplace transform of $K_{1}(t)$,

$$
\hat{f}(p)=4 \lambda_{0} \sum_{n=0}^{n_{F}} \frac{1}{p t_{T}+2(2 n+1)}
$$

$\left(n_{F}=\beta E_{F} / 2 \pi=k_{F} L_{T} / 4\right), K(t)$ is given by the inverse Laplace transform

$$
K(t)=\frac{1}{2 \pi i} \int_{-i \infty}^{+i \infty} d p e^{+p t} \ln [1+\hat{f}(p)] \simeq \frac{1}{\lambda_{0} \ln \left(k_{F} L^{*}\right)} K_{1}(t) \quad ; \quad L^{*}=\min \left(v_{F} t, L_{T} / 4\right) .
$$

The last equality is valid when $\ln k_{F} L^{*} \gg 1$ which is certainly satisfied when $\ln k_{F} l \gg 1$. Therefore, the higher-order terms merely lead to a renormalization of the coupling constant, thus reducing the predicted magnitude of the persistent current. In the high temperature regime $\left(L_{T} \ll L_{m}\right)$ the coupling constant is renormalized to $1 / \ln \left(k_{F} L_{T} / 4\right)$. Introducing $L_{m}=v_{F}(m L)^{2} / 4 D$, the average length of a trajectory diffusing $m$ times around the ring, one gets at low temperature $\left(L_{T} \gg L_{m}\right)$ a replacement of $\lambda_{0} \equiv 1$ by $1 / \ln \left(k_{F} L_{m}\right)$. These two limits agree with results obtained diagrammatically by Eckern [16].

We note that the functional form of the temperature dependence (exponential $T$ damping [15]) is in line with experiments [3 [5] while the amplitude of the persistent current with renormalized coupling constant is smaller than the experiments by a factor of $\sim 5$.

\section{E. Diffusive two-dimensional systems}

Contrary to rings, the geometry imposes no shortest length for returning paths in singlyconnected systems. One therefore expects a different temperature dependence of the magnetic response.

Consider a two-dimensional singly-connected diffusive quantum dot. In view of the general renormalization property of diffusive systems Eq. (19), the diagonal part of the thermodynamic potential from the entire Cooper series [Eq. (10)] can be written as

$$
\Omega^{(D)}=\frac{1}{\beta} \int d \mathbf{r} \int d t \frac{1}{\ln \left(k_{F} v_{F} t\right)} \frac{t_{T}}{t^{2}} R^{2}\left(\frac{t}{t_{T}}\right) \mathcal{A}(\mathbf{r}, t ; B) .
$$

Here we have used $L^{*}=v_{F} t$ in (19) since the $R^{2}$ factor ensures that the main contribution to the integral comes from $t<t_{T}$. In two dimensions the conditional return probability, entering into $\mathcal{A}$, is conveniently expressed in terms of the Fourier transform [7]

$$
P(\mathbf{r}, \mathbf{r}, t \mid A)=\frac{1}{4 \pi^{2}} \int d k|k| e^{i k A} \frac{\exp (-|k| D t)}{1-\exp (-2|k| D t)}
$$

from which one obtains 


$$
\mathcal{A}(\mathbf{r}, t ; B)=\frac{1}{4 \pi D} \frac{R\left(t / t_{B}\right)}{t} .
$$

Here we introduced the magnetic time

$$
t_{B}=\frac{\phi_{0}}{4 \pi B D}=\frac{L_{B}^{2}}{4 \pi D} .
$$

It is related to the square of the magnetic length $L_{B}^{2}$ which denotes the area enclosing one flux quantum (assuming diffusive dynamics). Note that the function $R$ in Eq. (22) has a different origin than in Eq. (20).

Using the expression (22) in Eq. (20) and taking the second derivative with respect to the field, we find for the susceptibility,

$$
\frac{\chi^{(D)}}{\left|\chi_{\mathrm{L}}\right|}=-\frac{6}{\pi}\left(k_{F} l\right) \int_{\tau_{e l}}^{\infty} \frac{d t}{t \ln \left(k_{F} v_{F} t\right)} R^{2}\left(\frac{t}{t_{T}}\right) R^{\prime \prime}\left(\frac{t}{t_{B}}\right)
$$

where $R^{\prime \prime}$ is the second derivative of $R$. The susceptibility is normalized to the twodimensional diamagnetic Landau susceptibility $\chi_{\mathrm{L}}=-e^{2} /\left(12 \pi m c^{2}\right)$.

In the above time integral the elastic scattering time $\tau_{e l}=l / v_{F}$ enters as a lower bound. This cutoff must be introduced since for backscattered paths with times shorter than $\tau_{e l}$ the diffusion approximation (21) no longer holds [20]. On the other hand Eq. (24) holds true only as long as the upper cutoff time $t^{*} \equiv \min \left(t_{T}, t_{B}\right)$ is smaller than the Thouless time $t_{c}=L^{2} / D$ (with $L$ being the system size). For times larger than $t_{c}$ the dynamics begins to behave ergodically, and the two-dimensional diffusion approximation is no longer valid. Assuming $t^{*}<t_{c}$, Eq. (24) can be approximately evaluated by replacing $R\left(t / t_{T}\right)$ and $R^{\prime \prime}\left(t / t_{B}\right)$ by $R(0)=1$ and $R^{\prime \prime}(0)=-1 / 3$, respectively, and introducing the upper cutoff $t^{*}$ in the integral. The remaining integral yields for $t^{*} \gg \tau_{e l}$

$$
\int_{\tau_{e l}}^{t^{*}} \frac{d t}{t \ln \left(k_{F} v_{F} t\right)}=\ln \left\{\frac{\ln \left[k_{F} v_{F} \min \left(t_{T}, t_{B}\right)\right]}{\ln \left(k_{F} l\right)}\right\} .
$$

The $\log$-log form produced by the $1 / t \ln t$ dependence results from the wide distribution of path-lengths in the system- there are flux-enclosing paths with lengths ranging from about $v_{F} \tau_{e l}$ up to $v_{F} t^{*}$. In contrast, in the ring geometry discussed in the previous section the temperature dependence is exponential because the minimum length of flux-enclosing trajectories is the circumference.

The averaged susceptibility of a diffusive two-dimensional structure then reads

$$
\frac{\chi^{(D)}}{\left|\chi_{\mathrm{L}}\right|} \simeq \frac{2}{\pi}\left(k_{F} l\right) \ln \left\{\frac{\ln \left[k_{F} v_{F} \min \left(t_{T}, t_{B}\right)\right]}{\ln \left(k_{F} l\right)}\right\} .
$$

One thus finds a log-log temperature dependence for $t_{T}<t_{B}$ and a $\log -\log B$ dependence for $t_{T}>t_{B}$. With regard to magnitude, the magnetic response of diffusive systems is paramagnetic and enhanced by a factor $k_{F} l$ compared to the clean Landau susceptibility $\chi_{\mathrm{L}}$.

Eq. (26) agrees with results from Aslamazov and Larkin [12], Altshuler, Aronov and Zyuzin [13,14, and Oh, Zyuzin and Serota [17] obtained with quantum diagrammatic perturbation theory. The equivalence between the semiclassical and quantum approaches to diffusive systems may be traced back to the fact that the "quantum" diagrammatic perturbation theory relies on the use of the small parameter $1 / k_{F} l$ which can be viewed as a semiclassical approximation. 


\section{F. Conclusion}

To conclude, we developed a semiclassical approach to evaluate the interaction contribution of the grand potential in a high density perturbative expansion. We showed that the averaged quantum magnetic response can be expressed in terms of an operator containing the classical probability for particles to return. As an application we computed the orbital magnetic response of diffusive rings and two-dimensional quantum dots arising from the combined effects of disorder and interaction.

RAJ and KR acknowledge support from the French-German program PROCOPE. The Division de Physique Théorique is "Unité de recherche des Universités Paris 11 et Paris 6 associée au C.N.R.S.".

[1] Electron-electron interactions in Disordered systems, edited by A.L. Efros and M. Pollak (North-Holland, Amsterdam, 1985).

[2] U. Sivan, R. Berkovits, Y. Aloni, O. Prus, A. Auerbach, and G. Ben-Yoseph, Phys. Rev. Lett. 77, 1123 (1996); O. Agam, N.S. Wingreen, B.L. Altshuler, D.C. Ralph, and M. Tinkham, Phys. Rev. Lett. 78, 1956 (1997).

[3] L.P. Lévy, G. Dolan, J. Dunsmuir, and H. Bouchiat, Phys. Rev. Lett. 64, 2074 (1990).

[4] V. Chandrasekhar, R.A. Webb, M.J. Brady, M.B. Ketchen, W.J. Gallagher, and A. Kleinsasser, Phys. Rev. Lett. 67, 3578 (1991).

[5] P. Mohanty, E.M.Q. Jariwala, M.B. Ketchen, and R.A. Webb, in Quantum Coherence and Decoherence, edited by K. Fujikawa and Y.A. Ono (Elsevier, 1996).

[6] For recent reviews see, e.g. U. Eckern and P. Schwab, Adv. Phys. 44, 387 (1995); K. Efetov, Supersymmetry in Disorder and Chaos, Cambridge University Press (1996).

[7] N. Argaman, Y. Imry, and U. Smilansky, Phys. Rev. B 47, 4440 (1993).

[8] F. von Oppen and E.K. Riedel, Phys. Rev. B 48, 9170 (1993); F. von Oppen, Phys. Rev. B 50, 17151 (1994).

[9] D. Ullmo, K. Richter, and R.A. Jalabert, Phys. Rev. Lett. 74, 383 (1995); K. Richter, D. Ullmo and R.A. Jalabert, Phys. Rep. 276, 1 (1996).

[10] G. Montambaux, Journal de Physique 6, 1 (1996).

[11] D. Ullmo, H.U. Baranger, K. Richter, F. von Oppen, and R.A. Jalabert, Report No. cond-mat/9708092 and unpublished.

[12] L.G. Aslamazov and A.I. Larkin, Sov. Phys.-JETP 40, 321 (1975).

[13] B.L Altshuler, A.G. Aronov, and A.Yu. Zyuzin, Sov. Phys.-JETP 57, 889 (1983).

[14] For a review see B.L. Altshuler and A.G. Aronov in Ref. [1].

[15] V. Ambegaokar and U. Eckern, Phys. Rev. Lett. 65, 381 (1990).

[16] U. Eckern, Z. Phys. B 42, 389 (1991).

[17] S. Oh, A.Yu. Zyuzin, and R.A. Serota, Phys. Rev. B 44, 8858 (1991).

[18] A.A. Abrikosov, L.P. Gorkov, and I.E. Dzyaloshinski, Methods of Quantum Field Theory in Statistical Physics (Prentice-Hall, Englewood Clifs, 1963).

[19] Chaos and Quantum Physics, edited by M.-J. Giannoni, A. Voros, and J. Zinn-Justin (North-Holland, New York, 1991).

[20] Shorter paths with $t<\tau_{e l}$ arise from higher order interaction events and contribute to the clean bulk magnetic response which however is negligible compared to the disorder induced interaction contribution [1]]. 